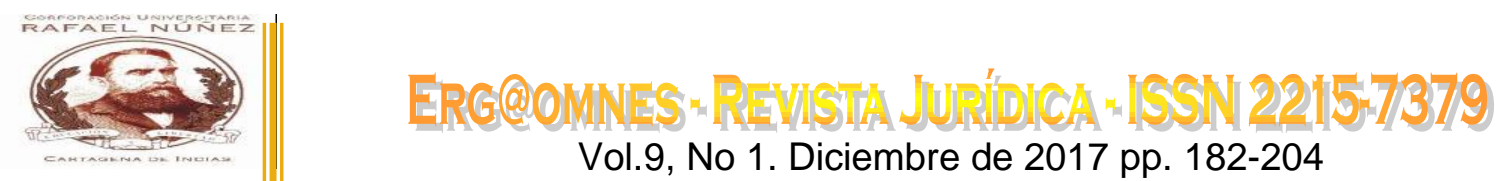

Artículo Original

Recibido para publicación: Julio 30 de 2017

Aceptado para publicación: Noviembre 1 de 2017

\title{
Ensayo del proceso de auto acreditación de una institución ${ }^{1}$.
}

\section{Test of the self-accreditation process of an institution.}

Autor: Iliana Marcela Fortich Lozano. ${ }^{2}$

Correspondencia: ilianamarcelafortich@hotmail.com.

\begin{abstract}
RESUMEN
Este artículo se escribe con el propósito de trasmitir los conocimientos sobre el proceso de auto acreditación que cada institución educativa de nivel superior debe desarrollar; para obtener la calidad de los programas que la universidad ofrece. El proceso de acreditación que realiza AFEIDAL a las Facultades de Derecho de Latinoamérica; es realizado con el objetivo, de progresar continuamente el propósito de desarrollo de la Universidad frente a los retos sociales, económicos y culturales que se dan firmemente en nuestra colectividad latinoamericana en el siglo XXI. No obstante, enfocado desde la perspectiva del Derecho como objeto de evaluación, por el perfil de la asociación y por su trascendencia principal dentro de la sociedad.
\end{abstract}

\section{Palabras Claves:}

Autoevaluación, progreso, educación superior, programa de derecho, currículos actualizados.

\begin{abstract}
This article is written with the purpose of transmitting the knowledge about the process of self-accreditation that each higher education institution must develop; To obtain the quality of the programs that the university offers. The accreditation process carried out by AFEIDAL to the Law Faculties of Latin America; Is carried out with the objective of continuously advancing the University's development goal in the face of the social, economic and cultural challenges that are firmly established in our Latin American community in the XXI century. Nevertheless, it is focused from the perspective of the Law as an object of evaluation, by the profile of the association and by its main transcendence within the society.
\end{abstract}

\section{Keywords:}

${ }^{1}$ Este artículo es producto de lo aprendido en el curso-taller de Formación de Responsables del Proceso de Auto Acreditación de cada Institución, bajo los principios, documentos, parámetros, criterios e indicadores, elaborados y aprobados por el Instituto Internacional de Acreditación del Derecho (IIDEA), A.C., bajo los auspicios de la Universidad de San Carlos de Guatemala y del Colegio de Santo Tomas, con el propósito de actualizarse sobre los procesos de Auto Acreditación de las facultades de Derecho de Colombia.

2 Docente Universidad Libre Sede Cartagena, Corporación Universitaria Rafael Núñez CURN, Especialista en Seguridad Social Universidad de Cartagena, Candidata a Magister en Derecho Universidad de Cartagena, Abogada Corporación Universitaria de la Costa CUC. Email: ilianamarcelafortich@hotmail.com. 
Self-assessment, progress, higher education, law program, updated curricula.

\section{Introducción}

El proceso de acreditación y autoevaluación de las universidades como forma de vigilancia de su desarrollo ha sido de gran importancia. Ya que, no solo se intenta nivelar los errores existentes en los distintos programas académicos a nivel mundial, sino que también corresponde a la interacción firme de las diferentes corporaciones para una aportación bilateral de sus desiguales trabajos con el propósito de desarrollarse de manera paralela.

Son garantías para los estudiantes en los diferentes planteles educativos la realización de intercambio entre universidades y sirve como estrategia principal para velar por un papel importante de la universidad para el servicio hacia la sociedad. Pero para ello, como primer paso se debe verificar los avances de cada programa académico y de las cátedras que se imparten. Además de los aportes significativos que podían brindarse en medio de esa interacción constante.

Así como los países desarrollados practican los intercambios universitarios, Latinoamérica también tenía que apropiarse de esas ideas y participar de este mismo desarrollo interuniversitario en donde se busca el adelanto de la academia como producto para el servicio a la sociedad. Ya que, el siglo XXI está inmerso en muchos desafíos sociales y requiere de una educación de calidad, con grandes transformaciones en materia de flexibilización, de multiculturalidad, de integralidad y de desarrollo del futuro del continente, para desarrollar el personal necesario del futuro de Latinoamérica con competencias eficaces y con perfiles de gran valoración, actitud y firmeza frente a los nuevos problemas que están por venir. Es importante tener personal preparado en los temas de autoevaluación y auto acreditación dentro de las Universidades Latinoamericanas. 
Desde esta perspectiva, mirando el contexto actual las facultades de derecho desarrollaron la asociación AFEIDAL, y el Instituto Internacional de Acreditación del Derecho (IIDEA, A.C), con el propósito de cumplir los designios de la acreditación de los diferentes programas de derecho existentes en la américa latina. AFEIDAL ofrece este servicio con el objetivo de asegurar el progreso entre las facultades de derecho y con la intención de garantizarle un reconocimiento a nivel continental que valore el papel que desempeñan las facultades para la comunidad contextual, además que también contribuyen a la academia y desarrollan personal calificado.

Por ello, el papel de AFEIDAL mediante la acreditación de los programas es garantizar la transparencia del funcionamiento de los programas de derecho para tener un mejor acceso a la educación y tener facultades de alta calidad en escala global.

Pero todo el sistema de acreditación que se maneja en este organismo supraestatal, se desarrolla con las bases de acreditar a los programas acorde con las necesidades del continente americano con el fin de alcanzar niveles educativos fuertes y profesionales reconocidos internacionalmente pero acorde con lo que se desarrolla en América Latina. Sin socavar lo que se esté presentando a nivel mundial, ya que de igual forma, paralelo al desarrollo de las acreditaciones y evaluaciones de los programas existentes en las facultades de derecho de nuestro continente, siempre se tiene puesto un ojo al futuro y a lo que sucede globalmente.

\section{Metodología.}

El presente trabajo fue de corte descriptivo - pedagógico, con método inductivo en el que se buscó reproducir sucintamente los aprendizajes del curso-taller de Formación de Responsables del Proceso de Auto Acreditación de cada Institución, bajo los principios, documentos, parámetros, criterios e indicadores, elaborados y aprobados por 
el Instituto Internacional de Acreditación del Derecho (IIDEA), A.C., bajo los auspicios de la Universidad de San Carlos de Guatemala y del Colegio de Santo Tomas.

\section{Resultados y discusiones.}

\section{Diagrama de acreditación}

El diagrama de acreditación está conformado por organismos acreditadores, dichos organismos deben establecer unos criterios, parámetros, instrumentos e indicadores, medios con los cuales las universidades van hacer evaluadas o medidas a través de la autoevaluación con la intervención de los visitadores evaluadores.

\subsection{Criterios de calidad.}

Los criterios de calidad se establecen teniendo en cuenta que la institución educativa superior cuente con la contratación de los profesores determinando que un treinta por ciento sean profesores jóvenes que otro treinta por ciento sea de edad media y el otro treinta por ciento sean profesores que están próximamente a jubilarse. Este mecanismo de contratación garantizan un equilibro en la permanencia y retención de profesores.

Para determinar la idoneidad de los aspirantes al programa académico se deben establecer estrategias de contratación donde un alto número de docente sean de tiempo completo y un bajo número sean profesores catedráticos.

Para garantizar las calidad del programa también se deben tener en cuenta que se deben establecer mecanismos estrategias para detectar los alumnos que se encuentren en situación de riesgo y montar estrategias de atención. La calidad tiene que ver con la coherencia de los argumentos presentados; y la eficacia se demuestra a través de la aplicación de los recursos. La eficiencia se nota en un programa académico con el uso 
correcto de los recursos y las acciones realizadas reflejan un impacto favorable en la aplicación de la justicia; de la aplicación de la ley y el desempeño de las autoridades.

De igual forma la calidad también se nota en la oportunidad en la entrega de los recursos; pertenecía de los recursos y las acciones respecto de la necesidad. También se reflejan la calidad en la suficiencia del recurso relacionada con la realidad a cubrir, y por último para demostrar en su totalidad la calidad del programa también se refleja en la transparencia, en el manejo que se le da a los recursos.

Por otra parte una institución de educación superior debe mostrar certeza y confianza para los padres de familia de los estudiantes que harán parte de este plantel educativo acerca de la buena formación académica que recibirán los alumnos. Los padres de familia deben sentirse complacidos con la calidad que les proporciona la universidad, estos les da satisfacción al compartir con sus hijos los éxitos académicos obtenidos, de igual forma la calidad del programa también se acredita con el otorgamiento de becas estudiantiles para los estudiantes que obtengan un promedio establecido por la universidad.

Las universidades para reflejar su calidad también deben propender la posibilidad de continuar con un posgrado de calidad y ofrecer becas a los estudiantes sobresalientes. Se debe facilitar la celebración de convenios de colaboración con otras instituciones de educación superior tanto nacional como internacional. Proporcionando lo relatado anteriormente la universidad podrá contar con egresados que formen parte del conglomerado de profesionales calificados y logar insertarse más rápidamente en el mercado laboral.

Para los empleadores es de mayor seguridad recibir personal egresado de universidades con un programa de alta calidad y acreditado nacional $e$ internacionalmente; por esta razón estarán más seguros los empleadores en el crecimiento empresarial por las contribuciones de grupo de profesionales incorporados en la organización 


\section{Contenido del programa académico.}

El programa académico debe tener un equilibrio adecuado entre profesores antiguos y nuevos; con producción de material didáctico, publicación de libros, artículos científicos. Con experiencias en las actividades profesionales con un currículo actualizado y pertinente, con altas tasas de eficiencia terminal, retención de alumnos, egresados y titulados.

El programa académico debe ofrecer servicios eficientes y oportunos de atención individual y grupal de estudiantes que se enseñe los materiales que verdaderamente sean necesarios; y se lleve realice una estadística de eficiencia terminal en donde se determinen cuantos alumnos ingresan y cuantos se titulan realmente.

\subsection{Documentación necesaria para la autoevaluación de la institución.}

La autoevaluación debe ser colectiva con la participación de todo el personal administrativo; se debe contar con la participación de los estudiantes y de los egresados. El cuestionario de autoevaluación es el documento primordial para la realización de la misma, para contestar el cuestionario de autoevaluación no debe hacerse como un ejercicio administrativo sino que debe implementarse entre el personal que participa como una cultura permanente. Para la revisión del cuestionario de autoevaluación el primer criterio será la revisión de los indicadores mínimos en caso de $\mathrm{n}$ tener esta información se suspenderá el proceso hasta obtener los indicadores.

\subsection{Acciones que se deben realizar para la autoevaluación.}

1. Establecer una oficina responsable del proceso de autoevaluación; o una coordinación. 
2. Establecer los lineamientos, estrategias, acciones y responsabilidades.

3. Generar instrumentos de evaluación por parte de la institución.

4. Conservar el respaldo de evidencias tanto en documentos impresos como en formato digital.

5. Evidencias por departamento un responsable por cada carpeta.

6. El plan deberá difundirse a la comunidad universitaria, de manera escrita y mediante la página web de la facultad de derecho.

7. Todas las áreas del programa académico deberán ser asesoradas por el responsable.

8. Una vez generada la evidencia y complementada las acciones deberá aprobarse en forma definitiva por el órgano correspondiente de la institución.

9. Dar contestación al cuestionario de autoevaluación por medio del coordinador de acreditación, el auxiliar del coordinador y los asistentes que pueden ser los estudiantes.

Se debe establecer para que el proceso sea más eficiente; debemos construir la oficina de la recopilación de la información y la realización de la contratación con el Instituto de Acreditación IIDEA.

\subsection{Marco general del proceso.}

El marco general debe estar conformado por el cuerpo académico que es igual a la constitución de un grupo de profesores de tiempo completo de un programa académico que comparten una o varias líneas de generación de aplicación del conocimiento.

El cuestionario debe ser proporcionado por el Instituto de Acreditación IIDEA; se debe contar con el currículo actualizado de cada docente. 
Para el llenado del cuestionario debe representar un esfuerzo institucional un trabajo sostenido por varios meses sentido de manera colegiada y estar enlazados del mismo trabajo colegiado. Es recomendable que para dar respuesta a cada uno de los indicadores se realice una descripción lo más detallada posible, no calificando si se cumple o no con el indicador; también se recomienda adjuntar como evidencia además de los oficios donde las autoridades dan respuesta a la petición de información hay que adjuntar imágenes, documentos de trabajo o cualquier otra evidencia corresponda con el indicador. Recuerde que el destinatario debe afirmar y presentar evidencia; debe enviar el texto en Word o equivalente entre las ligera sea la información será mejor. Cuando sean documentos oficiales se recomienda que sean en versión de imágenes, documento completo y con menos peso. Los aspectos considerados relevantes así como los abordados parcialmente así como los no abordados se deben presentar a la inicio de la visita de verificación, las evidencias deben coincidir con el indicador; no hay que confiar en los procesadores de textos hay que revisar bien la ortografía del texto.

Para que se dé la eficiencia terminal debe existir una relación cuantitativa entre los alumnos que ingresan y los que egresan de una corte en un programa educativo. La eficiencia de titulación debe tener una proporción alumnos de una corte que logra obtener el título profesional reconocido por la autoridad competente.

Dentro del marco general del proceso se debe incorporar a los estudiantes en la participación de programas y proyectos institucionales; también se debe incluir la normatividad institucional es decir el conjunto de leyes, estatutos y reglamentos de la universidad. Se debe optar por la movilidad académica que es la posibilidad de que estudiantes y profesores pueden realizar actividades académicas en diferentes programas.

El perfil de ingreso está relacionado con las características que se requieren que tenga el aspirante al iniciar un programa educativo; y el perfil del egresado se 
determina con las competencias que deben reunir los estudiantes al culminar el programa educativo.

Dentro del marco general del proceso también se debe realizar las tutorías que es la forma de relación pedagógica que se establecen mediante diversas actividades entre el profesor y el estudiante, docente tutor-alumno tutorado.

La vinculación de una institución de educación superior se hace a través de una función sustantiva, la cual se relacionan con otras instituciones educativas superiores y los sectores social, público y privado del ámbito local, regional, nacional e internacional.

La visión del programa es la situación a la que aspira una institución o programa educativo.

El visitador evaluador par académico que previamente capacitado designe IIDEA para realizar las visitas de verificación para efecto de acreditación de los programas académicos de derecho que ofrecen las instituciones educativas superiores. Para la relación de cada visitas se deben contar con unos requisitos mínimos que son aquellos indicadores que implican condiciones esenciales de la institución educativa superior; también se exigen unos requisitos necesarios que vienen siendo indicadores básicos y por ultimo unos requisitos deseables.

\subsection{Indicadores de la evaluación.}

Se valoran en una escala entre (1-5), la calificación no se puede impugnar, solo cuando no se acredite la institución educativa superior. La calificación en cinco es cuando la institución educativa tiene un funcionamiento excelente, en cuatro es sobresaliente, en tres es suficiente, en dos insuficiente y en uno no existe la evidencia. 
La información que se está trabajando debe estar guardada en varios computadores, memorias y correo electrónico colocar fecha y hora a cada documento grabado; la evidencia debe guardarse tanto en fisco como en medio electrónico.

Para la realización de la visita el par evaluador debe tener en cuenta el código de ética al iniciar y al terminar la visita. Es importante que el equipo multidisciplinario sepa manejar la información para poder llenar correctamente el formulario.

El término del proceso es aproximadamente de seis meses contando desde la realización de la visita hasta el concepto final.

\subsection{Actividades que debe realizarse antes de la visita.}

1. Los visitadores deben revisar el documento, las evidencias y acordar la logística del proceso.

2. El coordinador se contacta con los pares evaluadores para acordar la agenda.

3. El visitador revisa el cuestionario.

4. Anota las dudas e identifica las autoridades.

5. Organiza la visita.

6. Preparar la lista de invitados para la entrevista de egresados, empleadores, profesores, estudiantes y autoridades.

7. Conocer y manejar el código de ética.

8. Revisar y ajustar la agenda.

Revitalizar el proceso académico es la tarea del par evaluador externo.

\subsection{Actividades durante la visita.}


1. Los evaluadores participan como parte del equipo del programa académico y dan sugerencias para mejorarlo.

2. Hay que conocer y aplicar el código de ética, cuidar que se cumpla la agenda en tiempo.

3. Generar un ambiente de respeto, trabajar solo la jornada oficial.

4. El evaluador no deberá de dar nada por supuesto, y debe responder solo lo que se le pregunte.

5. Evitar conflictos innecesarios.

6. Preparar los tiempos y espacios para las entrevistas.

7. Se debe recordar que el cuestionario contempla los indicadores cruzados.

8. Participar en resolver las dudas con el equipo visitador.

\subsection{Actividades después de la visita.}

1. Reunión del equipo responsable con los participantes del proceso de autoevaluación.

2. Retroalimentar el proceso como si se sintiera logros alcanzados.

3. Informar a la comunidad académica del proceso y expectativas de los resultados.

4. Reconocer a los participantes y agradecer a los egresados.

\subsection{Reuniones que se deben realizar para el planeamiento de las actividades dentro de la visita.}

Se deben realizar tres reuniones en la primera reunión se explican los requisitos mínimos del proceso y las metas que se esperan alcanzar en cada reunión; las reuniones no deben extenderse más de una hora en esta primera reuniones se realizaran los avances del proceso. Se revisara el trabajo colegiado (tutorías), se elaboraran las evidencias y se describirá la complejidad del proceso. 
En la segunda reunión se revisan detenidamente los documentos; se revisan los avances del proceso, el cuestionario y se brindaran recomendaciones.

Se revisan los responsables y los avances del proceso y se realiza un simulacro del acto de la autoevaluación.

\subsection{Organización del programa}

Con respecto a la organización del programa se debe tener en cuenta una serie de aspectos a evaluar:

1. que posea un plan de desarrollo vigente, con metas adquiribles y desde distintos rangos de tiempo.

2. el programa debe poseer un dirigente con grandes competencias y como mínimo debe ser abogado, con muy buen perfil académico, si es posible investigador, y con buenos títulos que le sirvan al programa para ser un buen dirigente.

3. el programa debe tener muy bien definido los cuerpos colegiados o distintos comités internos del programa, con sus funciones muy bien definidos y un orden de agenda muy bien estipulados para trabajar por el programa.

4. se debe tener bien sentado los perfiles de los profesores que le sirven a la institución superior y al programa.

5. también debe poseer un informe de autoevaluación antes del expuesto como requisito para AFEIDAL, donde sinceramente se demuestre avances de lo que con anterioridad era el programa.

Esta organización con el propósito de generar una idea de la estructura existente en el programa académico y de cómo se visualizan el mismo programa y la institución. Todo con el propósito de verificar un orden jerárquico y metodológico de lo existente en el programa. 


\subsection{Proceso educativo de los programas academicos de derecho}

Con respecto a los procesos educativos, si es necesario enviar una documentación que desarrolle todo lo concerniente al desarrollo de los conductos regulares, ya que eso evidencia la efectividad que desarrolla el programa y la eficiencia del personal de trabajo en la facultad de derecho, desde la perspectiva académica y administrativa. Por ello los documentos principales para evaluar serían los siguientes:

1. Se debe tener copia del plan de estudios de cada asignatura, en donde este contenido la malla curricular, la estrategia pedagógica utilizada, las diferentes actividades a utilizar en el semestre, el número de horas a utilizar de teoría y de prácticas de la materia, la forma de evaluación, los créditos específicos que maneje cada materia y su fundamentación teórica.

De igual forma se deben completar otros requisitos de la malla curricular más generales como por lo menos el perfil de ingreso y de egreso que van a tener los estudiantes de derecho, concomitante con la carga académica de las materias.

2. debe tenerse no solo la autoevaluación del programa en sí, sino también la revisión del plan existente en la actualidad por el programa.

3. especificar en un documento las modalidades $u$ optativas de titulación existentes, dependiendo si lo posibilita el programa, incluyendo la normatividad existente de ello, con el amparo de la institución superior a cargo.

Estos documentos ayudaran a verificar a AFEIDAL la calidad del personal que se desempeña en la institución de educación superior.

\subsection{Alumnos}


Con respecto a los alumnos, se puede considerar que es uno de los componentes principales, ya que ellos son el producto finalizado del servicio que prestan las instituciones de educación superior y sobre todo de las facultades de derecho de Latinoamérica, ya que ellos van a adquirir trabajos de gran responsabilidad social. Para evaluar este componente se debe de tener la siguiente documentación con requisitos específicos como:

1. normativas y requisitos de ingreso, muy bien redactado, para evitar confusiones.

2. normativas y requisitos de permanencia, muy detallado para no generar malas interpretaciones.

3. normativas y requisitos de egreso, muy bien explicado.

4. explicación de los índices de deserción y reprobación de las materias. Llevar las estadísticas anuales de estos fenómenos.

5. especificar los requisitos mínimos de admisión y detallar el proceso de selección, indicando un balance de por lo menos 3 años de anterioridad.

6. se debe tener un documento acerca del seguimiento de egresados y su impacto en la sociedad, dependiendo de los contrastes existentes tanto en el contexto como lo enseñado en la malla curricular.

Estos detalles documentales nos dan un mayor bosquejo del producto finalizado y su incidencia social, ya que de igual forma existe un seguimiento constante de los estudiantes que se convertirán en profesionales de alta calidad, adecuados a los contextos políticos y sociales.

\subsection{Profesores}

Con respecto a los profesores es perentorio tener un listado de la planta docente existente en la institución que contengan las calidades que contribuirán a que se consolide un buen producto final, o sea, que velaran por que los estudiantes sean de 
altas competencias para la sociedad, por eso es importante que envíen los siguientes documentos:

1. Categóricamente sus grados académicos y sus características contractuales con la institución de educación superior, además de otros detalles que complementen su perfil y su vinculación laboral.

2. También debe haber un documento que detalle de los profesores sus requisitos de ingreso a la institución superior de educación, así como también sus requisitos de permanencia estímulos y promociones salariales, como incentivo ya sea para el desarrollo académico o investigativo que contribuya a la educación superior.

3. Debe de existir un documento que explique la forma de ascenso de los profesores, con toda la normatividad y el debido procedimiento.

4. Debe existir también un documento donde se explique el personal de apoyo de los docentes.

Todo este seguimiento junto con las actividades que desarrollaran los pares evaluadores nos dan un bosquejo de la calidad del programa de derecho que se esta evaluando.

\subsection{Educación continúa}

Dentro del programa de derecho la institución de educación superior debe tener otros programas de educación continua que apoyen a la formación específica de los profesionales que se construyen para la sociedad. Obviamente que estos programas de educación continua tiene que tener un propósito fundamental para impartirlo que sirva para la comunidad. Ya que esto junto los postgrados existentes les garantizarían a la facultad un plus fundamental en la formación de los estudiantes y profesionales, y obviamente en la evaluación realizada por AFEIDAL. 
De igual forma los documentos importantes en la educación continua necesarios para su evaluación son:

1. la normatividad de todos los programas de Educación Continúa.

2. los números de cursos impartidos con la fundamentación de la temática.

3. cursos patrocinados con otras instituciones y con qué fin se realizó dicho curso.

\subsection{Investigación y desarrollo}

Con respecto al área de investigación es fundamental tener unos parámetros no solo desde la cosmovisión de la institución de educación superior sino también desde la perspectiva del país con respecto al pensamiento investigativo y al apoyo que ejerzan sobre procesos de estudios investigativos. Por ello, deben existir varios documentos específicos sobre esta temática que nos dan una luz acerca del componente filosófico de esta categoría como:

1. Un documento que explique las políticas de la investigación con toda la reglamentación al respecto. Incluyendo los parámetros para proyectos y resultados de investigaciones.

2. Un documento que detalle las principales líneas de investigación existentes en el programa y que también exponga los diferentes programas existentes para el impulso a la investigación, indicando la participación activa de los profesores y específicamente que campo académico explotan o que temáticas específicas investigan.

3. Un listado de las publicaciones existentes como productos de investigación y detallar si existe alguna revista científica en el programa de derecho.

4. Los recursos financieros destinados a la investigación con la reglamentación pertinente para ello. 
Esto nos detalla sobremanera la visión de la investigación de los programas de derechos de las facultades a evaluar y la importancia que le dan a la profundización en temáticas socio jurídicas, para el desarrollo de la sociedad. Es perentorio afirmar que la asociación es muy celosa en enfatizar en esta categoría, ya que las nuevas tendencias de la academia están enfatizadas en desarrollar la investigación a nivel mundial.

\subsection{Postgrados}

Los programas académicos que tengan postgrados deberán tener la siguiente información detallada:

1. un documento que contengan todos los planes de estudio con sus reglamentos, número de alumnos en los diferentes programas, el número de ingresos y egresos del programa de postgrados y si existen programas de becas o incentivos a la educación.

2. deben documentar la lista de profesores que participan en estos programas de postgrados señalando su grado académico, la modalidad de tiempo que desempeña en el programa y la calidad de la contratación con la institución. De igual forma se debe señalar los profesores externos con los requisitos antes mencionados, y por ultimo si existen convenios con otra institución en el cual se desarrolle ese postgrado.

3. señalar si el postgrado se encuentra en registrado en el ministerio correspondiente con permiso del país en el cual se ejerza. Donde se evidencia la normativa de aprobación del postgrado.

\subsection{Infraestructura}


Con respecto a la infraestructura física, es importante resaltarla, ya que este tipo de documentos nos detallan la logística existente para brindar cómodamente un servicio educativo de calidad, no obstante no puede dejarse de percibir que cualquier institución con falta de comodidades para implementar el servicio no va a rendir de igual forma a otra que posea los privilegios mínimos para desarrollar la educación profesional, esto no quiere decir que tiene que ser tecnológicamente la mejor, sino que debe tener algunos recursos físicos que permitan el desarrollo de la educación con una comodidad mínima, tanto para los profesores y los alumnos, ad portas de la modernidad.

Para esto se deben de documentar los siguientes ítems de infraestructura:

a. la existencia cuantitativa de las aulas de clase y las capacidades de alumnos.

b. los diferentes laboratorios con equipos específicos con los que cuentan.

c. auditorios o salas de conferencias, para realizar eventos o seminarios alemanes.

d. Oficinas para los docentes.

e. bibliotecas y espacios para la lectura.

f. espacios cibernéticos y los diferentes programas de internet ofrecidos para uso de las tecnologías en la academia.

g. equipos audiovisuales.

Estos elementos físicos mínimamente ayudan a desarrollar la educación con alta calidad y siendo proporcional al número de personas que se encuentran en el programa de derecho.

\subsection{Finanzas}


Es perentorio para el desarrollo del programa académico, manejar sus finanzas de manera que administrativamente pueda rendir a todas las divisiones de trabajo existentes.

Por ello debe presentar documentos que contengan:

1. el presupuesto anual utilizado en el programa y sus usos específicos.

2. un documento que contenga las fuentes de financiación específica, no solo de la institución superior, sino de las diferentes opciones de ingreso que tenga el programa.

3. un documento que explique detalladamente las políticas del uso del presupuesto y de cómo redefinirlo dependiendo del contexto.

\subsection{Vinculación y servicios a la comunidad}

En pro de la función social que desempeña el programa de derecho, es preciso afirmar que puede tener programas especiales que trabajen directamente con las comunidades como formación práctica a los estudiantes de derecho. Por ello si existe tal programa se debe documentar de la siguiente manera:

1. Realizar un listado que indique los convenios que existan con otras organizaciones en beneficio de la comunidad.

2. este listado debe contener los servicios específicos y los potenciales beneficiarios de los servicios con las diferentes características que sirvan a la comunidad.

3. y por último los resultados alcanzados por estos distintos programas hacia la comunidad.

$Y$ con este último componente se realizan las evaluaciones necesarias para arrojar los primeros índices de evaluación y esperar la visita de los pares pertinentes para que 
verifiquen la información y desarrollen las actividades que los convenzan de las ventajas de acreditar dicho programa de derecho. (iidea, manual de acreditacion del instituto internacional de acreditacion del derecho, 2015)

\section{De los visitadores}

Algunas de las funciones principales de los visitadores podrían desarrollarse entre las actividades que desempeñan en su ejercicio de evaluador del programa académico, pero la asociación AFEIDAL expone algunas de estas funciones las cuales son:

a. recibir y verificar toda la documentación del programa de derecho a evaluar.

b. verificar holísticamente durante la visita dicha información, si se cumple en la realidad a cabalidad o poseen falencias que le permitan identificar bien las categorías académico administrativas del programa a evaluar.

c. elaborar un informe para el comité técnico de evaluación y acreditación que contengan las fortalezas y criticas del programa evaluado.

d. informar sobre los procedimientos que se llevaran a cabo para la respectiva acreditación y evaluación del programa.

e. solicitar todo el acervo probatorio de lo contenido en la documentación oficial enviada para dicha evaluación.

f. realizar todas las entrevistas necesarias para evidenciar el avance del programa de derecho que se está evaluando.

\subsection{Perfil de los visitadores}

Los visitadores o pares de evaluación de un programa de derecho de una facultad latinoamericana, deben contener unos requisitos que le permitan evaluar con neutralidad 
y con alta calidad junto con el conocimiento necesario para realizar dicha labor. Estos requisitos son los siguientes:

a. contar con una licenciatura en el área de derecho y con grados de postgrados que le permitan tener un perfil de aceptación valido. Además de poseer como experiencia 3 años de gestión educativa en un programa de derecho de cualquier facultad acreditada por la asociación.

b. capacidad pedagógica acreditada con participación en proyectos académicos o de investigación.

c. conocimiento en informática y sistemas de información computarizada.

d. conocimiento de diversas modalidades educativas, el cual le brinde una alta gama de conocimiento especializado de la academia.

e. haber participado en proyectos y programas institucionales de gestión educativa.

Estos documentos le garantizarían la calidad y pertinencia de la persona que evalúa un programa de institución superior.

\section{Indicadores}

Existen unos indicadores en la primera valoración de la documentación revisada del programa de derecho que está compuesta por tres indicadores:

1. indicador con requisitos mínimos: este indica que el programa de derecho para evaluar posee algunas condiciones esenciales, para no decir precarias para operar como facultad.

2. indicador con requisitos necesarios: este indica que en la facultad el componente operativo posee unas condiciones básicas, lo cual le permitiría tener lo necesario como facultad. 
3. y el tercer componente o requisito deseable: este indicador expone un grado de favorabilidad muy bueno para el programa de derecho, ya que posee condiciones óptimas de funcionamiento.

\section{Visita de los pares académicos}

La principal actividad después de verificar el documento de autoevaluación del programa de derecho, junto con toda la evidencia presentada es la visita de pares. Como lo habíamos desarrollado en las funciones, a los visitadores les corresponde identificar si la documentación enviada presenta con certeza todo lo estipulado. $Y$ todo esto lo desarrollaran organizando una serie de actividades en la institución de educación superior.

Las calificaciones las realizara cada evaluador de manera individual y con esto enviaran en el informe técnico que realicen de sus visitas una serie de recomendaciones para el mejoramiento de las condiciones del programa académico en derecho.

Toda la calificación la realizaran en el cuadernillo indicado por AFEIDAL para realizar la evaluación.

\section{Conclusiones}

Podemos concluir este artículo confirmando que el proceso de acreditación de un programa de derecho de cualquier facultad latinoamericana, no es menos exigente que lo que se exige para la acreditación de instituciones educativas superiores en los países desarrollados; este proceso ostenta todas las calidades para considerar el desarrollo equivalente de los programas en las instituciones de educación superior.

Se reflexiona que el proceso de auto acreditación está protegido de imparcialidad y experiencia necesaria para realizar dichos procesos, no solo por los evaluadores 
seleccionados y los seminarios, cursos, talleres que realiza AFEIDAL e IIDEA, sino por la exposición detallada de todos los mecanismos establecidos en un programa académico de derecho, y la experiencia de como se le señala positivamente para tener un desarrollo progresivo de alta calidad en las instituciones que se afilian y hacen parte de esta asociación y que pretenden estar acreditados por la misma.

La asociación no solo acredita, sino que es un entidad de apoyo para las falencias existentes en los programas de derecho latinoamericanos, ofrecidos por las universidades. Es de conocimiento público que esta asociación siempre estará dispuesta a colaborar para la unificación, la multiculturalidad y la internacionalización de lo que se está cimentando constantemente en américa latina.

Con la formación de esta asociación latinoamericana tan importante como lo ha demostrado con la realización de capacitaciones relacionadas con el tema de auto acreditación de las instituciones educativas superiores podremos lograr constituir una unión latinoamericana como la conformada en los países desarrollados y así mejoraremos la convivencia en nuestra sociedad.

\section{Referencias.}

2. iidea. (2015). manual de acreditacion del instituto internacional de acreditacion del derecho. mexico: iidea.

3. iidea. (2015). marco general del proceso de acreditacion del instituto internacional de acreditacion del derecho. mexico: iidea.

Carvajal Martínez, Jorge Enrique, \& Guzmán Rincón, Andrés Mauricio. (2017). Economía de mercado y democracia: elementos para una crítica al discurso del desarrollo promovido por las Instituciones Financieras Internacionales. Justicia, (31), 116-134. https://dx.doi.org/10.17081/just.22.31.2602 
Cialti, Pierre-Henri. (2016). La mayor representatividad sindical en España*. Justicia, (30), 17-31. https://dx.doi.org/10.17081/just.21.30.1347

Cárdenas, L. (2013). Prácticas de lectura y escritura en la universidad. ¿Qué y cómo leen y escriben los estudiantes de licenciaturas? Revista Amauta, 21, $139-162$

Cruz, E. (2015). la Ciudadanía ECológiCa En aBya-yala/ amériCa latina. Amauta, 87-103.

Echeverría, A. M. (2015). Derechos correlacionales a la justicia transicional, verdad, justicia y reparación. Erg@omnes, 7(1), 1-29. https://doi.org/10.22519/22157379.740

Gutiérrez Messino, C. (2014). Factores relacionados con el incumplimiento de la obligación alimentaria en hijos e hijas menores en Barranquilla 2014. Erg@omnes, 6(1), 1-16. https://doi.org/10.22519/22157379.449

Maury Palacio, N. (2014). Libre desarrollo de la personalidad en cambio de nombre a persona por segunda vez en Colombia. Erg@omnes, 6(1), 56-65. https://doi.org/10.22519/22157379.454

Mejía Cáez, Miguel Ramón. (2017). El derecho internacional de los derechos humanos, un nuevo concepto. Justicia, (32), 38-63. https://dx.doi.org/10.17081/just.23.32.2904

Mejía Turizo, J., Medina Solano, S., \& Carmona Vergara, H. (2014). Arbitrariedad en las decisiones judiciales y administrativas. Erg@omnes, 6(1), 150-164. https://doi.org/10.22519/22157379.460 
Pabón Arrieta, Juan Antonio, \& Torres Argüelles, Alfredo. (2017). La interpretación jurídica en el litigio estratégico en defensa de los derechos humanos en Colombia. Justicia, (32), 227-242. https://dx.doi.org/10.17081/just.22.32.2915

Ricaurte Villalobos, I. A. (2015). Cláusula de los derechos humanos en los tratados de libre comercio suscritos por Colombia. Erg@omnes, 7(1), 161-172. https://doi.org/10.22519/22157379.749

Roca Franco, J. F., \& Escobar Alcaraz, J. (2015). Doble periodo de vacaciones como derecho de carácter principal a trabajadores catalogados de alto riesgo. Erg@omnes, 7(1), 134-149. https://doi.org/10.22519/22157379.747

Rodríguez-Burgos, Karla, Martínez Cárdenas, Alán Alejandro, \& Rodríguez-Serpa, Ferney Asdrual. (2017). Estudio empírico sobre los valores democráticos de tolerancia y respeto en la generación milenaria. Justicia, (31), 135-150. https://dx.doi.org/10.17081/just.22.31.2603

Sanabria, Fabián. (2016). Estado, instituciones democráticas y postconflicto en Colombia*. Justicia, (30), 86-95. https://dx.doi.org/10.17081/just.21.30.1351 